\title{
Pharmacophore-based design of sphingosine 1-phosphate-3 receptor antagonists that include a 3,4-dialkoxybenzophenone scaffold
}

Yuuki Koide, Kazuhiro Uemoto, Takeshi Hasegawa, Tomoyuki Sada, Akira Murakami,

Hiroshi Takasugi, Atsuko Sakurai, Naoki Mochizuki, Atsuo Takahashi, Atsushi Nishida

\section{Contents:}

1. One hundred seven structures in database to assess the GH score.

2. Structures of 36 assayed compounds.

3. Elemental analysis data. 
1. One hundred seven structures in database to assess the GH score.

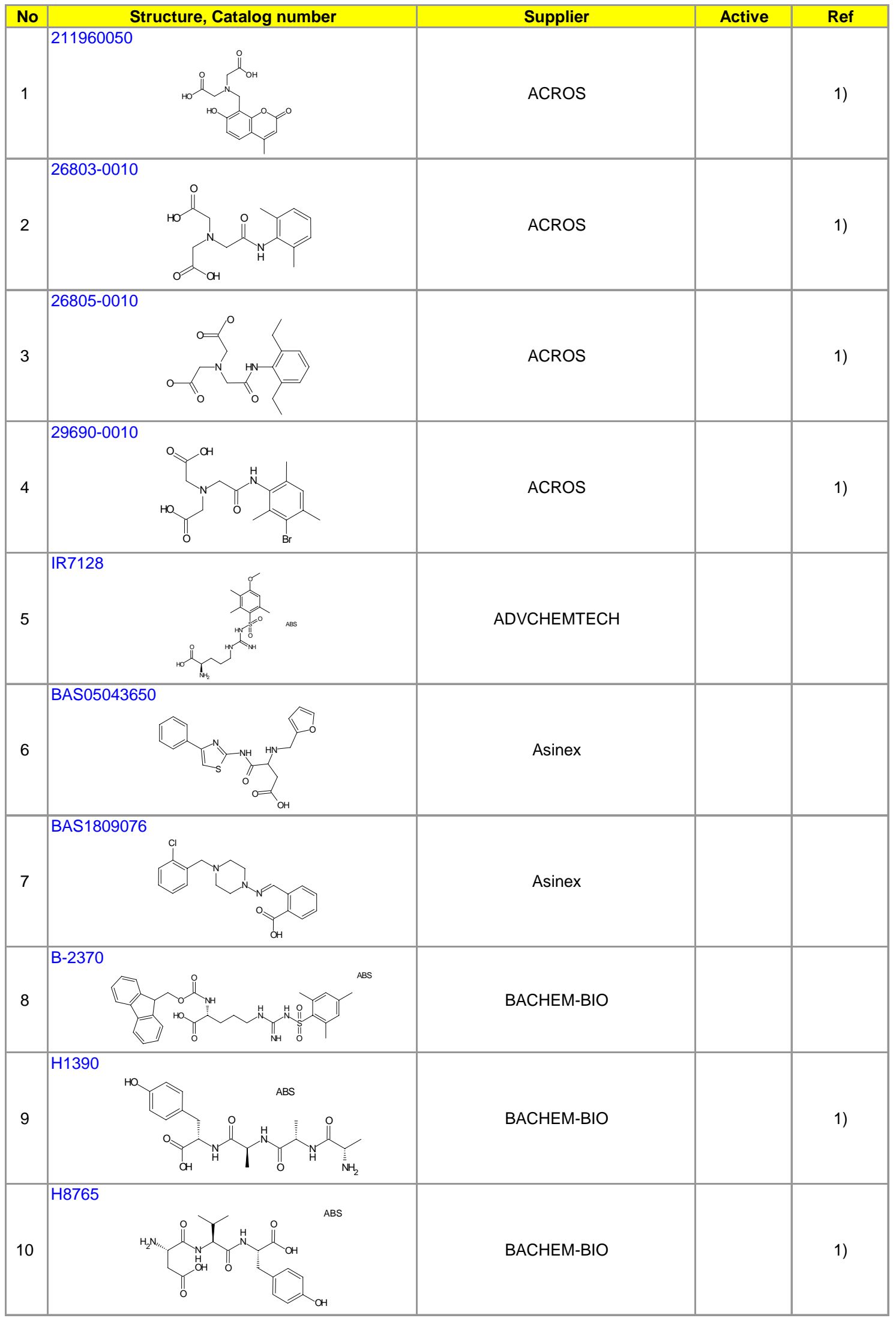




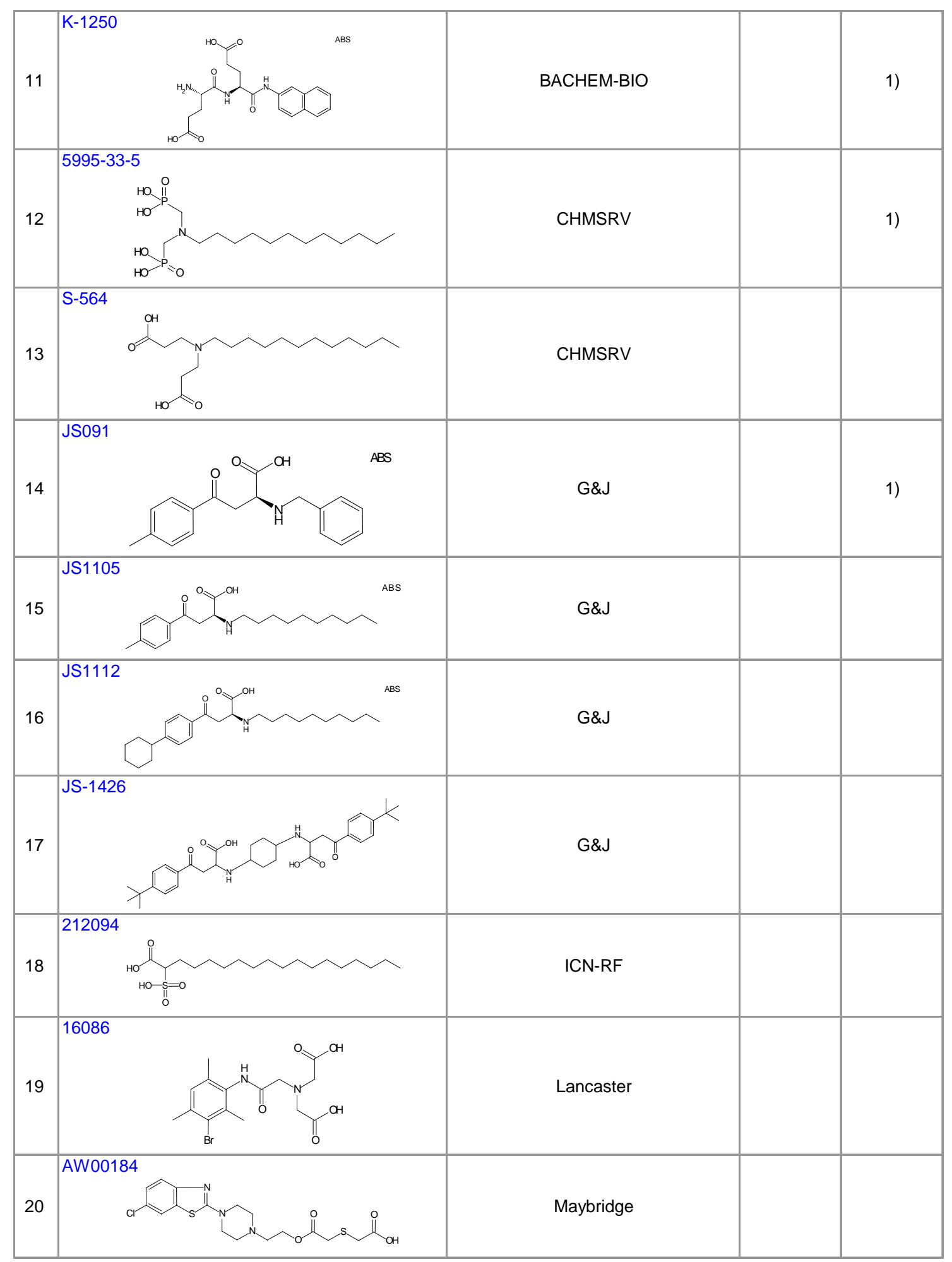




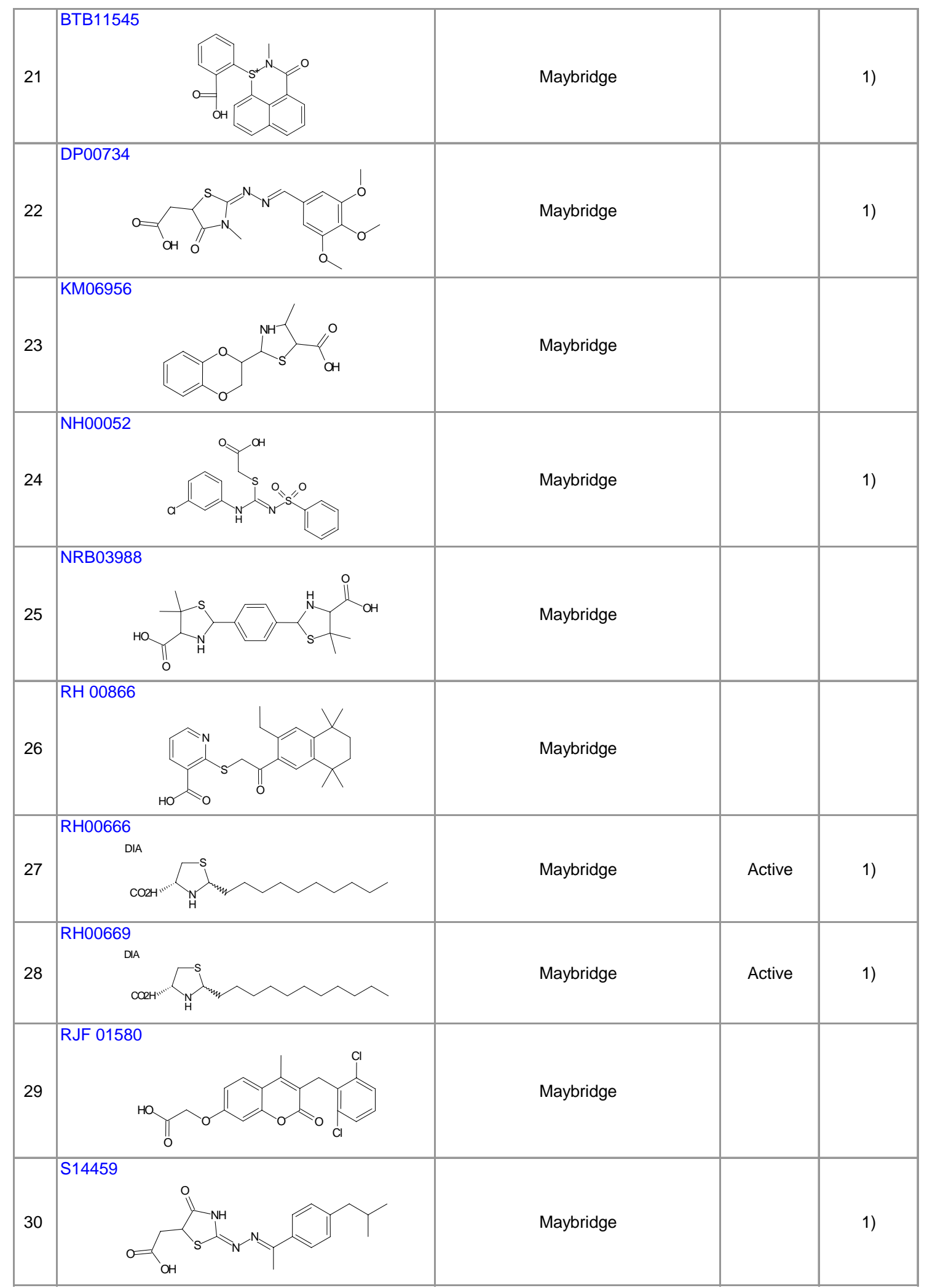




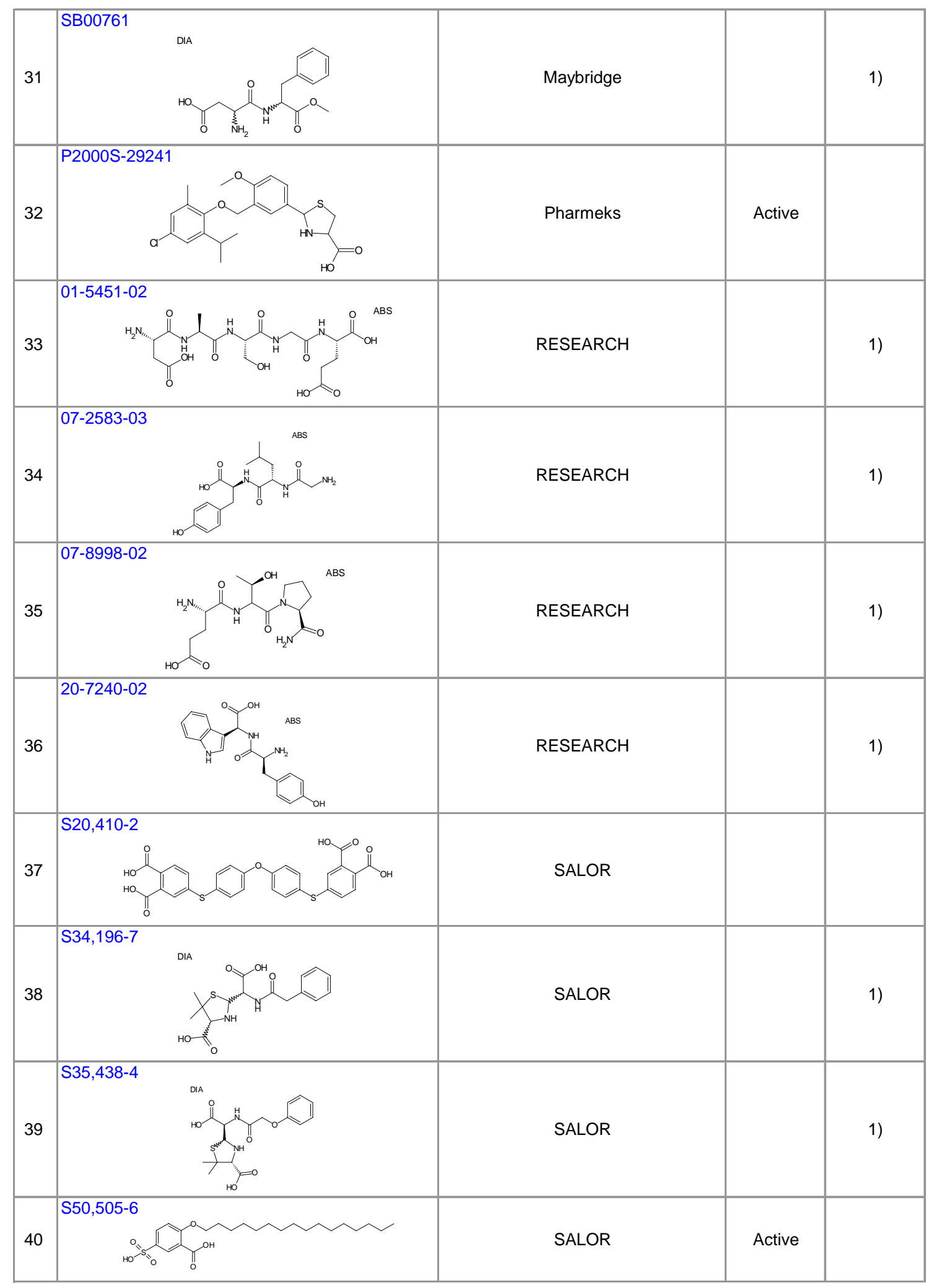




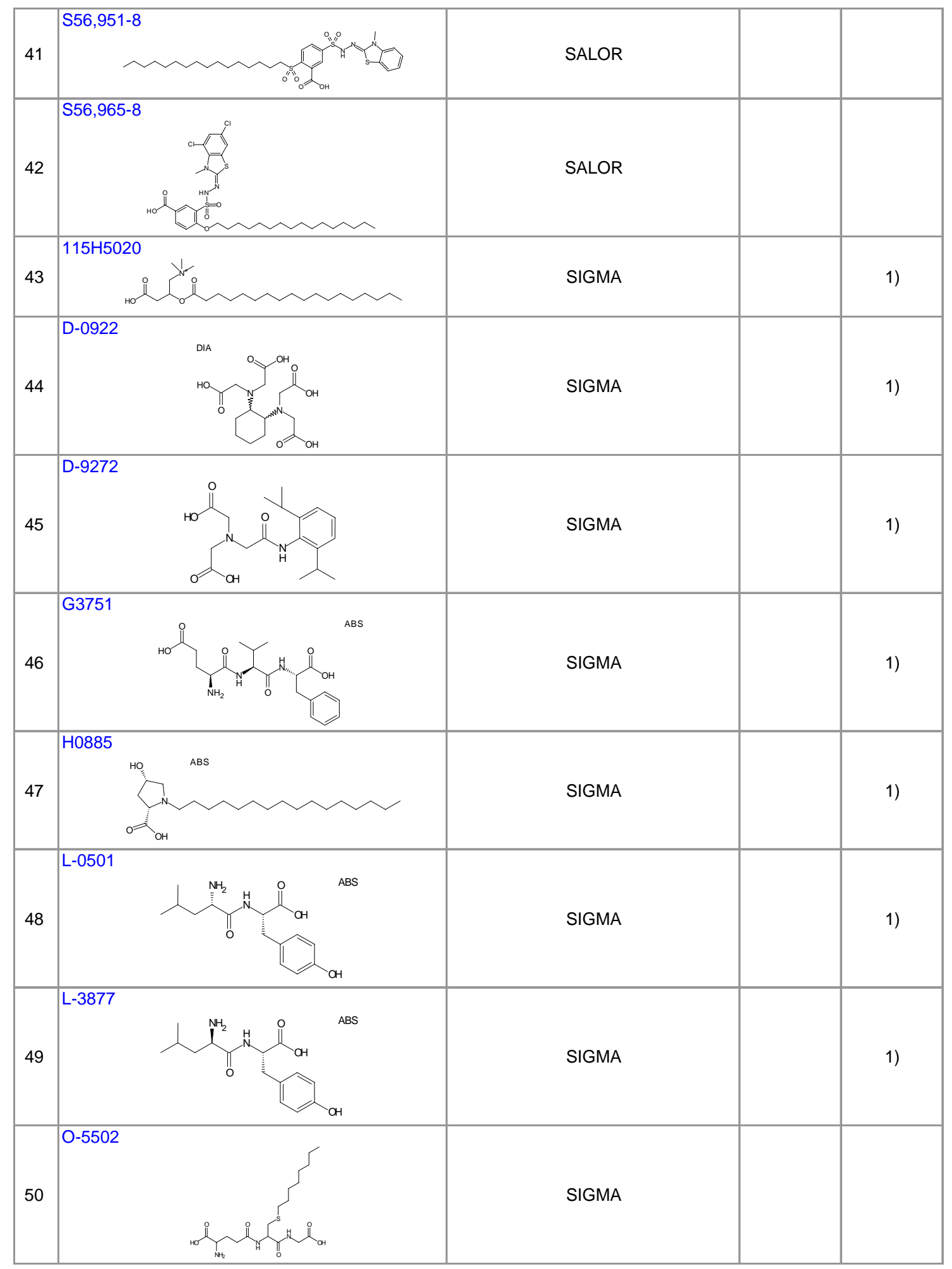




\begin{tabular}{|c|c|c|c|}
\hline 51 & P4509 & SIGMA & 1) \\
\hline 52 & T6779 & SIGMA & \\
\hline 53 & AE-641/14714112 & Specs & \\
\hline 54 & AE-848/07789007 & Specs & \\
\hline 55 & AE-848/31941058 & Specs & \\
\hline 56 & AE-848/37331006 & Specs & \\
\hline 57 & AF-399/34567055 & Specs & \\
\hline 58 & AF-826/30392019 & Specs & \\
\hline 59 & AF-962/31930018 & Specs & \\
\hline 60 & AG-205/37007316 & Specs & \\
\hline
\end{tabular}




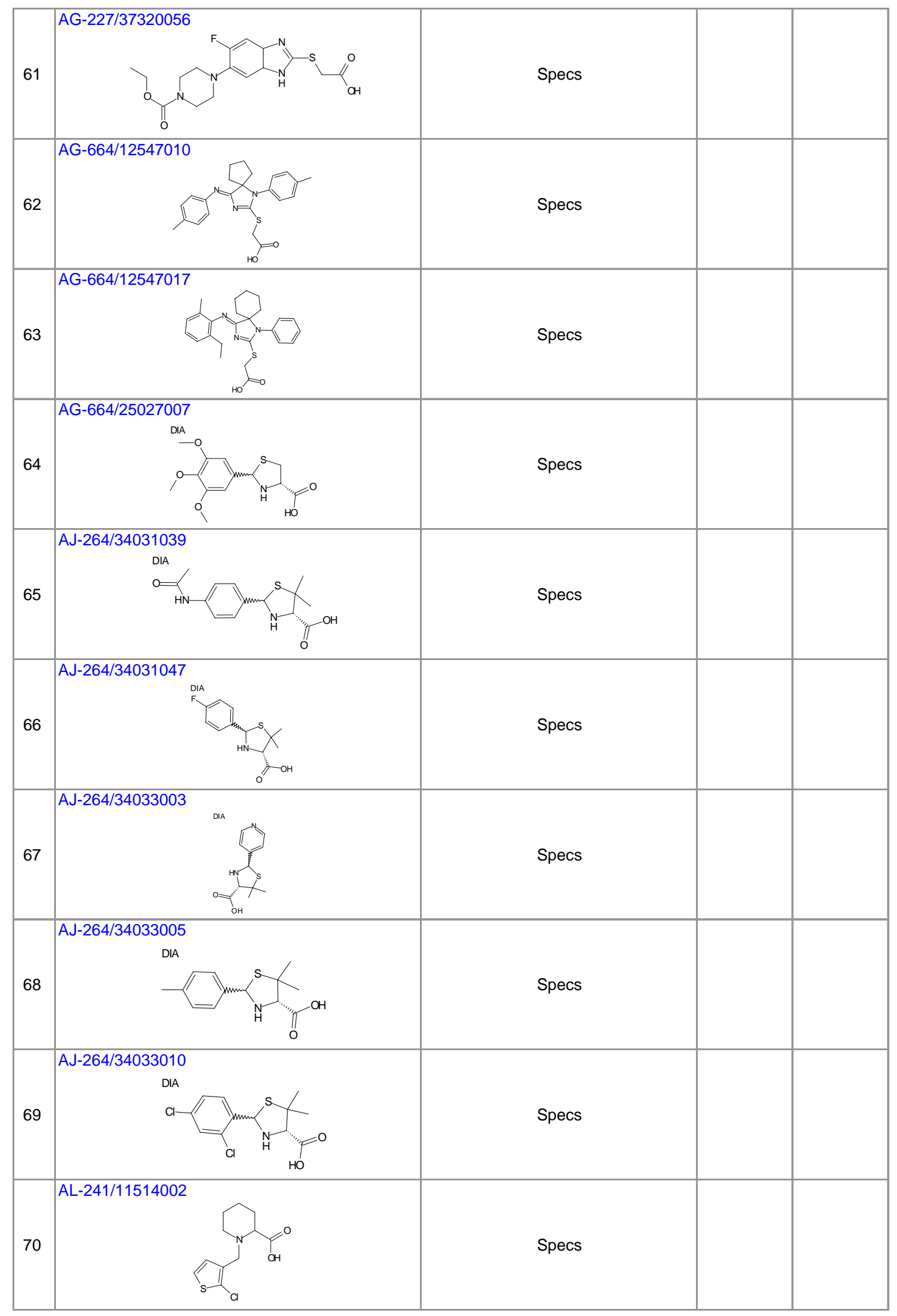




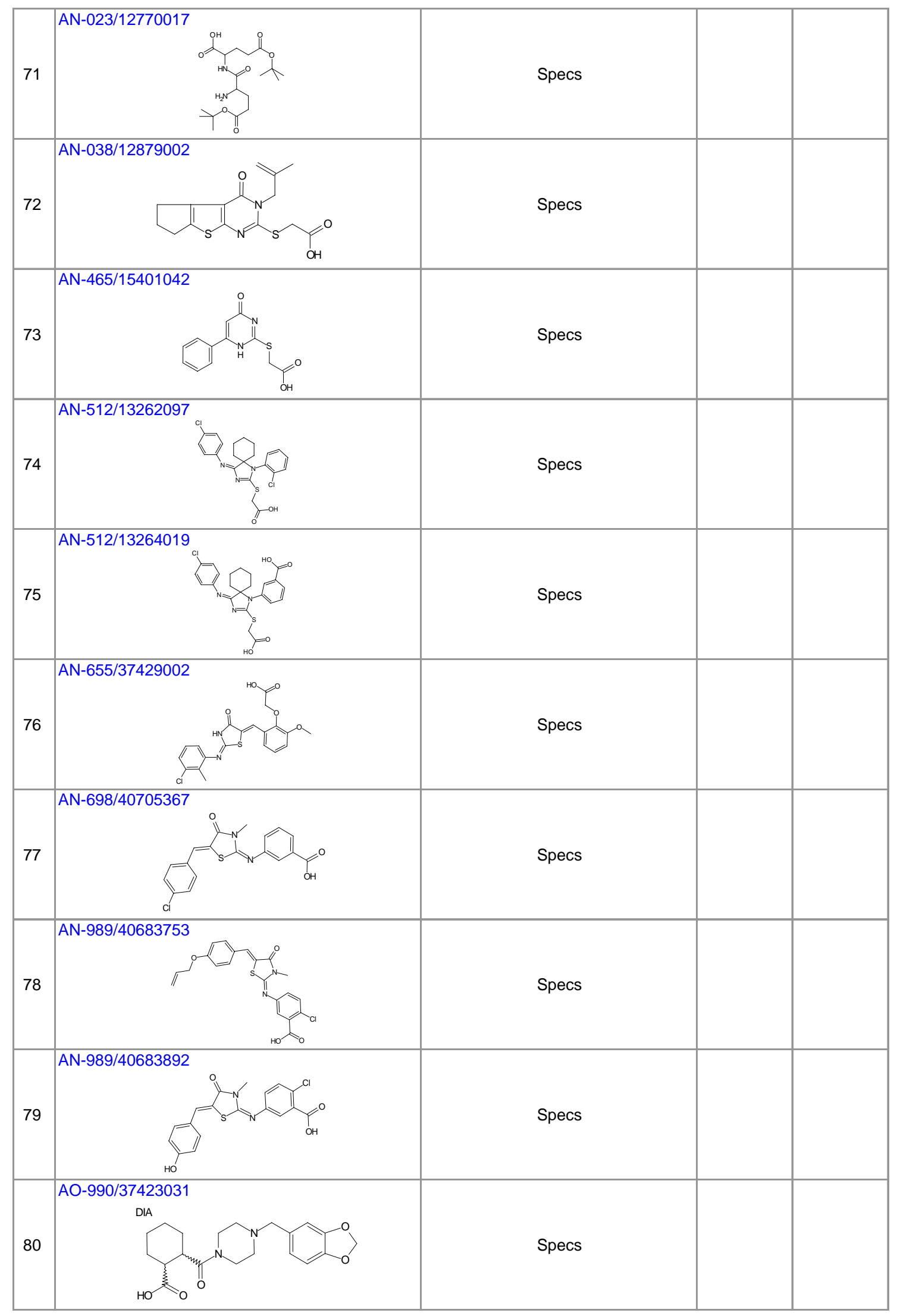




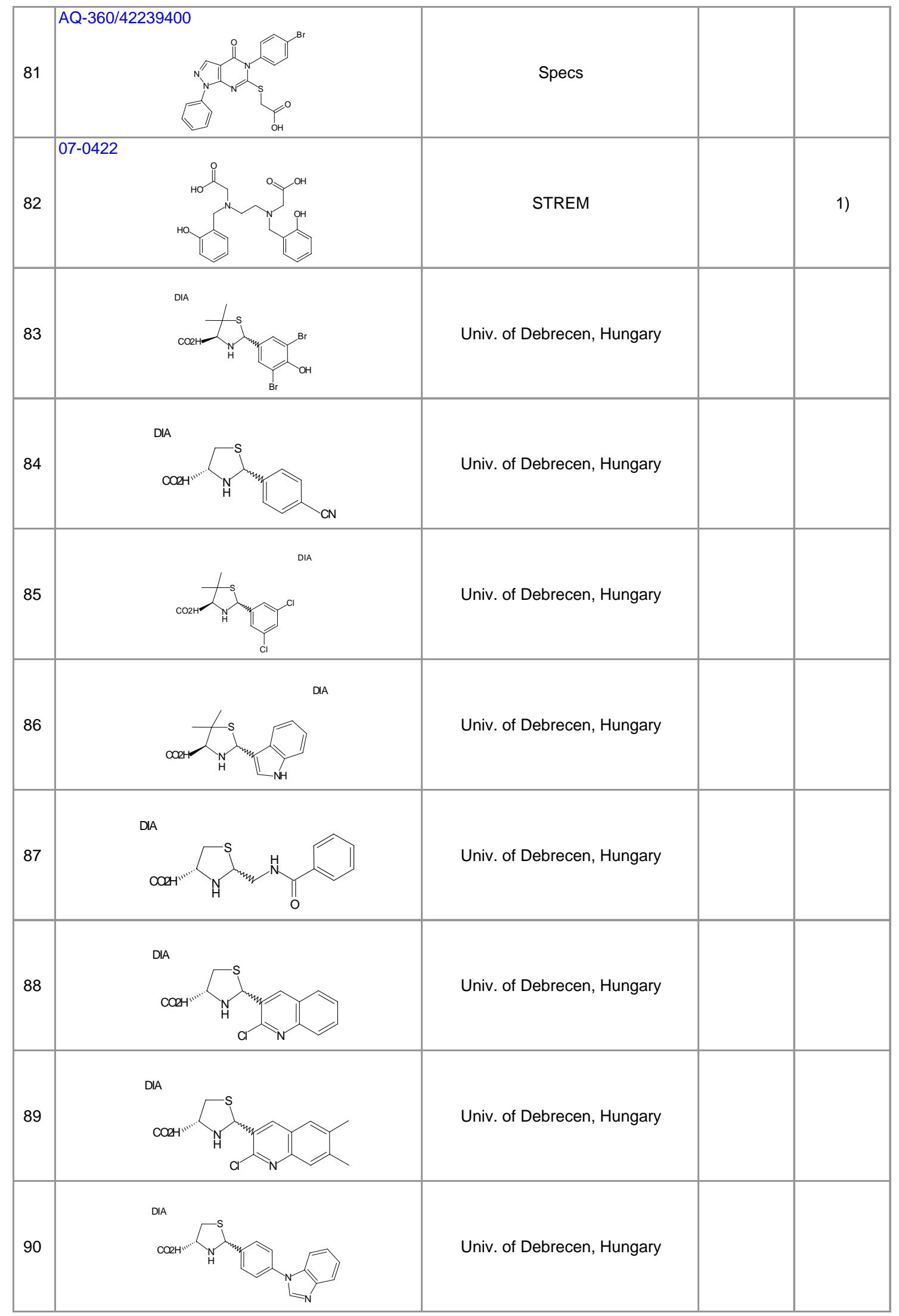




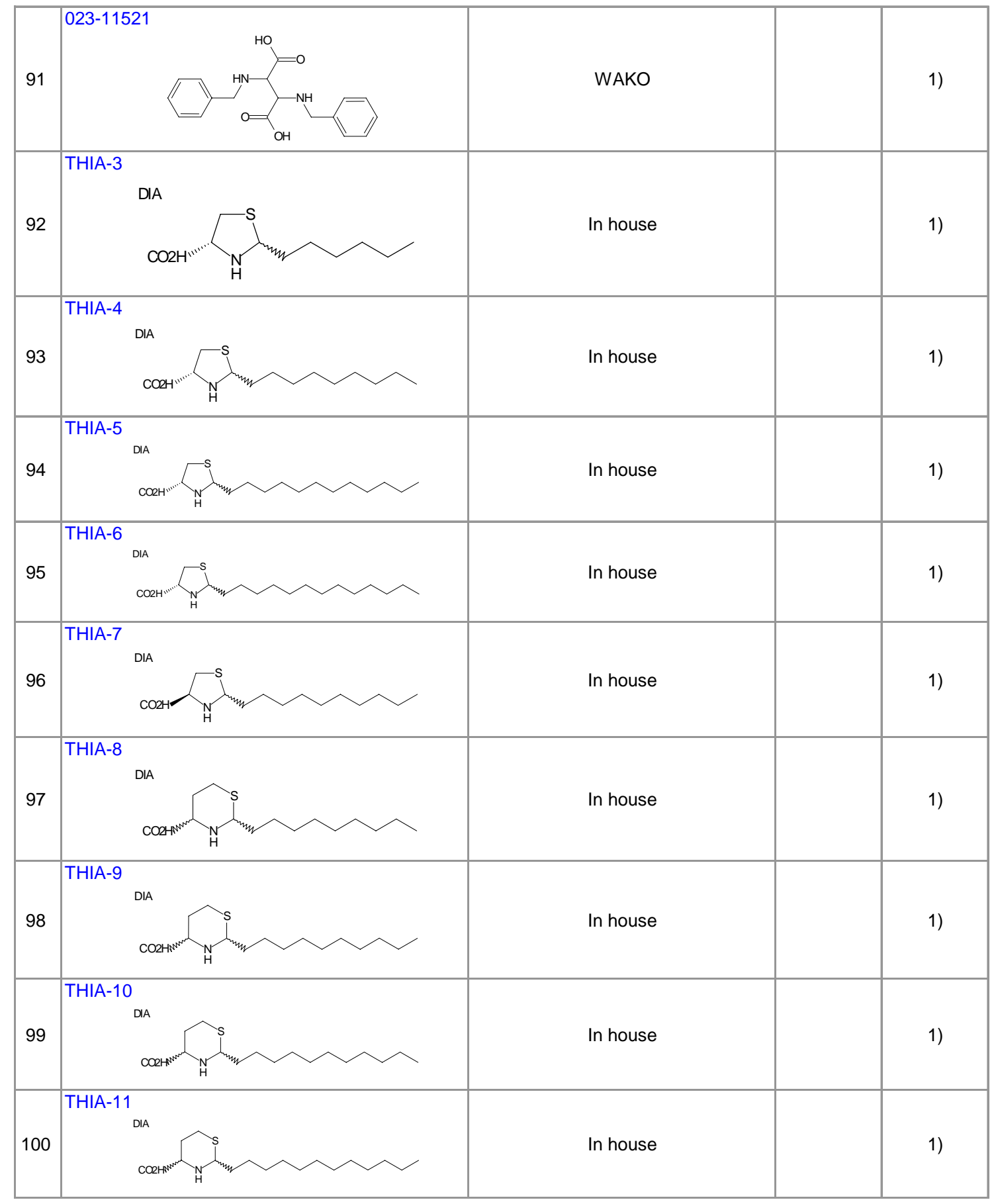




\begin{tabular}{|c|c|c|c|c|}
\hline 101 & $\begin{array}{r}\text { THIA-12 } \\
\text { DIA }\end{array}$ & In house & & 1) \\
\hline 102 & $\begin{array}{r}\text { THIA-19 } \\
\text { DIA }\end{array}$ & In house & & 1) \\
\hline 103 & $\begin{array}{r}\text { THIA-20 } \\
\text { DIA }\end{array}$ & In house & Active & 1) \\
\hline 104 & $\begin{array}{r}\text { THIA-21 } \\
\text { DIA }\end{array}$ & In house & Active & 1) \\
\hline 105 & $\begin{array}{r}\text { THIA-22 } \\
\text { DIA }\end{array}$ & In house & & 1) \\
\hline 106 & $\begin{array}{r}\text { THIA-23 } \\
\text { DIA }\end{array}$ & In house & Active & 1) \\
\hline 107 & THIA-24 & In house & & 1) \\
\hline
\end{tabular}

1. Koide, Y.; Hasegawa, T.; Takahashi, A.; Endo, A.; Mochizuki, N.; Nakagawa, M.; Nishida, A., Development of novel EDG3 antagonists using a 3D database search and their structure-activity relationships. J. Med. Chem. 2002, 45, (21), 4629-38. 
2. Structures of 36 assayed compounds.

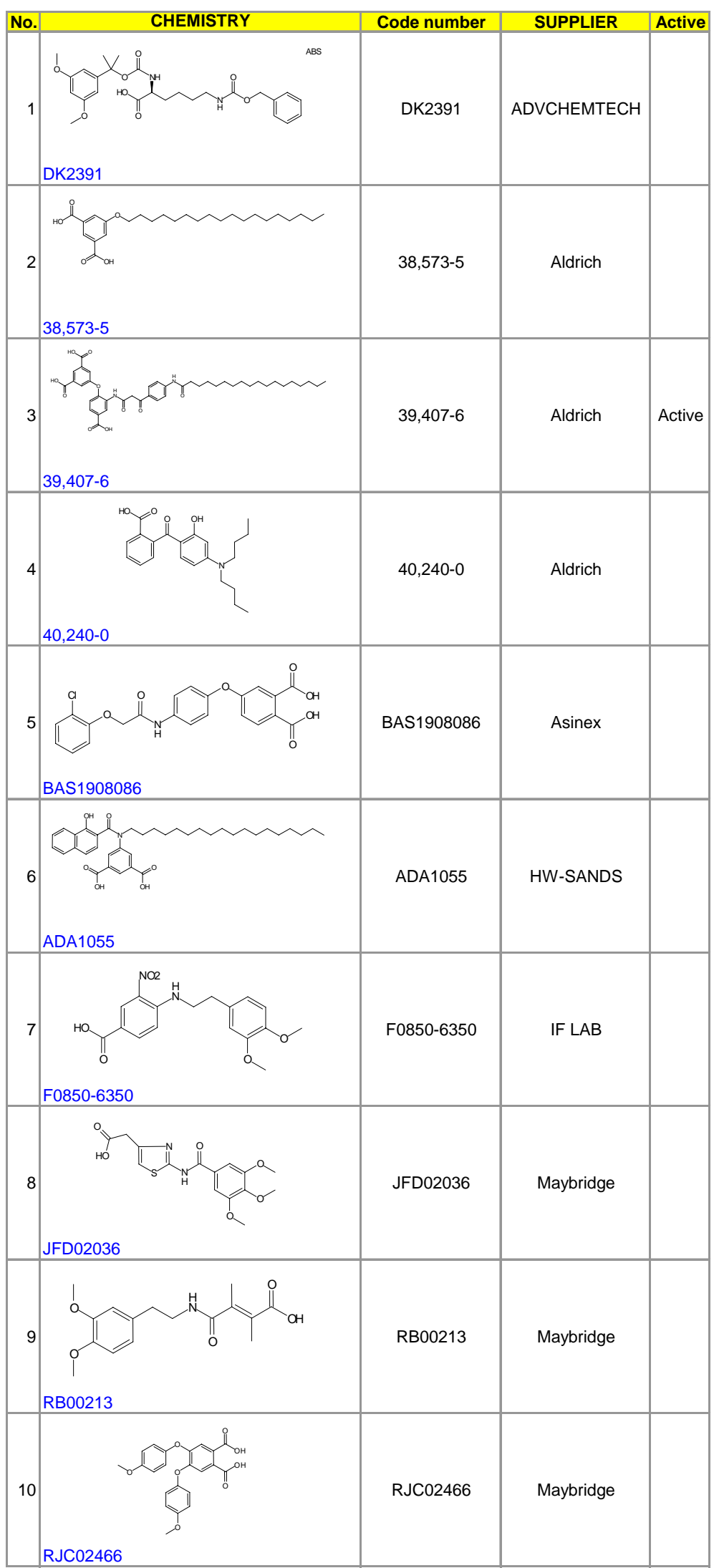




\begin{tabular}{|c|c|c|c|c|}
\hline 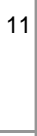 & SEW04756 & SEW04756 & Maybridge & \\
\hline 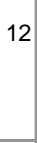 & $\begin{array}{c}-0 \\
\text { PFC-1462 }\end{array}$ & PFC-1462 & PEAKDALE & \\
\hline 13 & P2000S-083 & P2000S-08311 & Pharmeks & \\
\hline 14 & P2000S-099 & P2000S-09938 & Pharmeks & \\
\hline 15 & P2000S-2 & P2000S-26060 & Pharmeks & \\
\hline 16 & 2001S-0 & P2001S-046217 & Pharmeks & \\
\hline 17 & P2001S-07 & P2001S-076767 & Pharmeks & \\
\hline 18 & R24,209-8 & $R 24,209-8$ & SALOR & \\
\hline 19 & S033708 & S033708 & SALOR & Active \\
\hline 20 & S57,850-9 & S57,850-9 & SALOR & \\
\hline
\end{tabular}




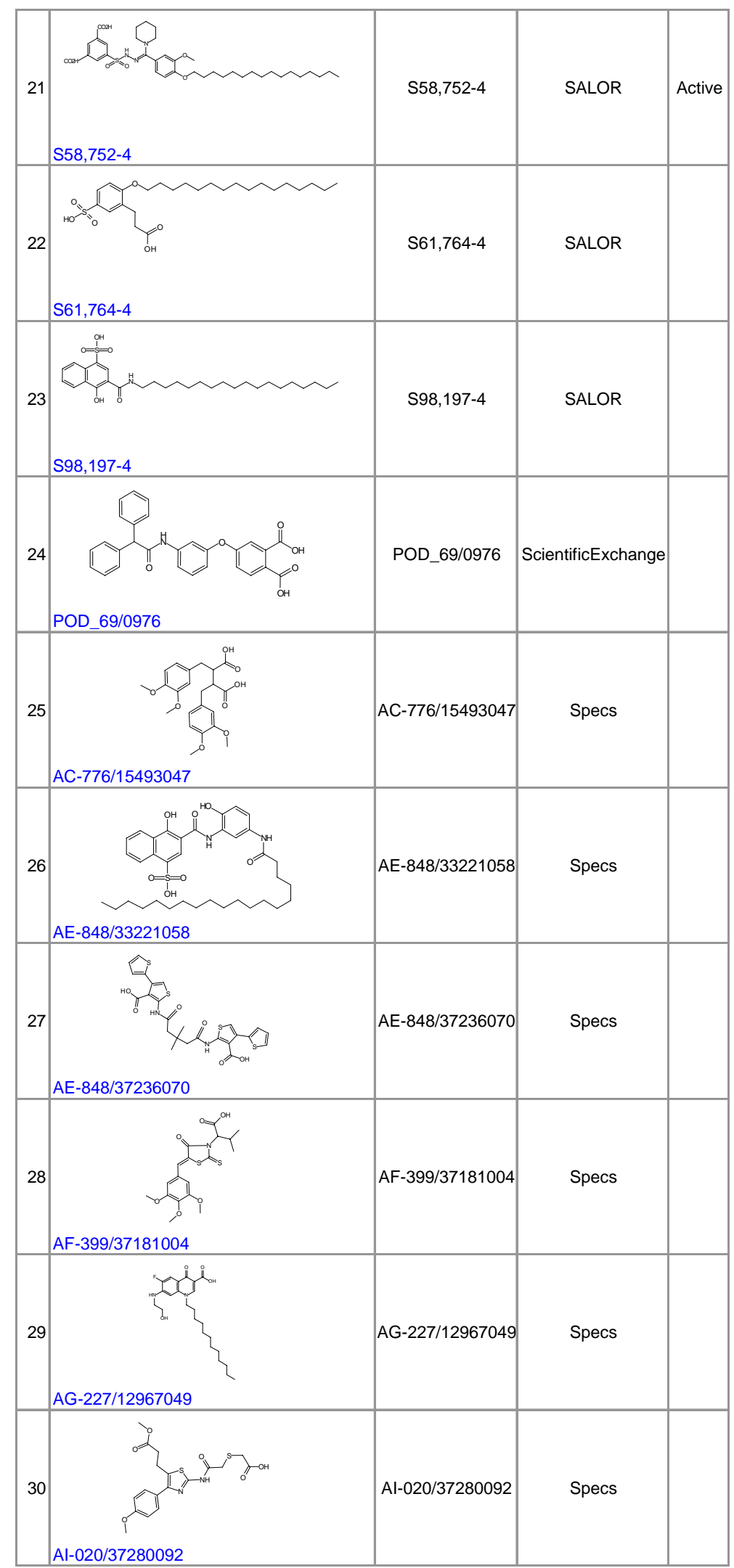




\begin{tabular}{|c|c|c|c|}
\hline 31 & AJ-091/33281060 & AJ-091/33281060 & Specs \\
\hline 32 & AN-652/13748003 & AN-652/13748003 & Specs \\
\hline 33 & AN-989/14072019 & AN-989/14072019 & Specs \\
\hline 34 & AP-064/15228987 & AP-064/15228987 & Specs \\
\hline 35 & AP-064/40657034 & AP-064/40657034 & Specs \\
\hline 36 & AP-372/40662 & AP-372/40662049 & Specs \\
\hline
\end{tabular}


3. Elemental analysis data.

\begin{tabular}{|c|c|c|c|c|c|c|c|}
\hline \multirow[b]{2}{*}{ Compound } & \multirow[b]{2}{*}{ Formula } & \multicolumn{3}{|c|}{ Calculated } & \multicolumn{3}{|c|}{ Found } \\
\hline & & $\mathrm{C}$ & $\mathrm{H}$ & $\mathrm{N}$ & $\mathrm{C}$ & $\mathrm{H}$ & $\mathrm{N}$ \\
\hline 17 & $\mathrm{C}_{10} \mathrm{H}_{9} \mathrm{ClO}_{6} \mathrm{~S}$ & 41.04 & 3.10 & & 41.35 & 3.10 & \\
\hline 19 & $\mathrm{C}_{10} \mathrm{H}_{12} \mathrm{~N}_{2} \mathrm{O}_{6} \mathrm{~S}$ & 41.66 & 4.20 & 9.72 & 41.72 & 4.09 & 9.55 \\
\hline 20 & $\mathrm{C}_{8} \mathrm{H}_{10} \mathrm{~N}_{2} \mathrm{O}_{4} \mathrm{~S}$ & 41.73 & 4.38 & 12.17 & 42.08 & 4.26 & 11.95 \\
\hline 21 & $\mathrm{C}_{30} \mathrm{H}_{44} \mathrm{O}_{3}$ & 79.60 & 9.80 & & 79.35 & 10.00 & \\
\hline 22 & $\mathrm{C}_{22} \mathrm{H}_{28} \mathrm{O}_{3}$ & 77.61 & 8.29 & & 77.39 & 8.41 & \\
\hline 23 & $\mathrm{C}_{18} \mathrm{H}_{20} \mathrm{O}_{3}$ & 76.03 & 7.09 & & 75.97 & 7.18 & \\
\hline 24 & $\mathrm{C}_{2} 9 \mathrm{H}_{42} \mathrm{O}_{2}$ & 82.41 & 10.02 & & 82.55 & 10.08 & \\
\hline $25 E$ & $\mathrm{C}_{40} \mathrm{H}_{54} \mathrm{~N}_{2} \mathrm{O}_{8} \mathrm{~S}$ & 66.46 & 7.53 & 3.87 & 66.45 & 7.53 & 3.89 \\
\hline $25 Z$ & $\mathrm{C}_{40} \mathrm{H}_{54} \mathrm{~N}_{2} \mathrm{O}_{8} \mathrm{~S}$ & 66.46 & 7.53 & 3.87 & 66.34 & 7.56 & 3.90 \\
\hline $26 E$ & $\mathrm{C}_{32} \mathrm{H}_{38} \mathrm{~N}_{2} \mathrm{O}_{8} \mathrm{~S}$ & 62.93 & 6.27 & 4.59 & 62.93 & 6.26 & 4.61 \\
\hline $26 Z$ & $\mathrm{C}_{32} \mathrm{H}_{38} \mathrm{~N}_{2} \mathrm{O}_{8} \mathrm{~S}$ & 62.93 & 6.27 & 4.59 & 62.64 & 6.10 & 4.40 \\
\hline $27 E$ & $\mathrm{C}_{28} \mathrm{H}_{30} \mathrm{~N}_{2} \mathrm{O}_{8} \mathrm{~S}$ & 60.64 & 5.45 & 5.05 & 60.74 & 5.34 & 5.27 \\
\hline $28 E$ & $\mathrm{C}_{3} 9 \mathrm{H}_{52} \mathrm{~N}_{2} \mathrm{O}_{7} \mathrm{~S}$ & 67.60 & 7.56 & 4.04 & 67.39 & 7.62 & 4.02 \\
\hline $29 E$ & $\mathrm{C}_{38} \mathrm{H}_{52} \mathrm{~N}_{2} \mathrm{O}_{6} \mathrm{~S}$ & 68.64 & 7.88 & 4.21 & 68.66 & 7.93 & 4.22 \\
\hline $29 Z$ & $\mathrm{C}_{38} \mathrm{H}_{52} \mathrm{~N}_{2} \mathrm{O}_{6} \mathrm{~S}$ & 68.64 & 7.88 & 4.21 & 68.73 & 7.99 & 4.05 \\
\hline $30 \mathrm{E}$ & $\mathrm{C}_{38} \mathrm{H}_{50} \mathrm{~N}_{2} \mathrm{O}_{8} \mathrm{~S}$ & 65.68 & 7.25 & 4.03 & 65.90 & 7.36 & 3.98 \\
\hline $30 \mathrm{Z}$ & $\mathrm{C}_{38} \mathrm{H}_{50} \mathrm{~N}_{2} \mathrm{O}_{8} \mathrm{~S}$ & 65.68 & 7.25 & 4.03 & 65.89 & 7.32 & 3.97 \\
\hline 31E & $\mathrm{C}_{30} \mathrm{H}_{34} \mathrm{~N}_{2} \mathrm{O}_{8} \mathrm{~S}$ & 61.84 & 5.88 & 4.81 & 62.00 & 5.88 & 4.68 \\
\hline $31 Z$ & $\mathrm{C}_{30} \mathrm{H}_{34} \mathrm{~N}_{2} \mathrm{O}_{8} \mathrm{~S}$ & 61.84 & 5.88 & 4.81 & 61.65 & 5.82 & 4.71 \\
\hline $32 E$ & $\mathrm{C}_{26} \mathrm{H}_{26} \mathrm{~N}_{2} \mathrm{O}_{8} \mathrm{~S}$ & 59.31 & 4.98 & 5.32 & 58.99 & 4.93 & 5.16 \\
\hline
\end{tabular}




\begin{tabular}{|c|c|ccc|ccc|}
\hline \multirow{2}{*}{ Compound } & \multirow{2}{*}{ Formula } & \multicolumn{3}{|c|}{ Calculated } & \multicolumn{3}{c|}{ Found } \\
\cline { 3 - 8 } & & $\mathrm{C}$ & $\mathrm{H}$ & $\mathrm{N}$ & $\mathrm{C}$ & $\mathrm{H}$ & $\mathrm{N}$ \\
\hline $33 \mathbf{3}$ & $\mathrm{C}_{37} \mathrm{H}_{48} \mathrm{~N}_{2} \mathrm{O}_{7} \mathrm{~S}$ & 66.84 & 7.28 & 4.21 & 66.73 & 7.50 & 4.29 \\
$34 \mathbf{3 4 Z}$ & $\mathrm{C}_{37} \mathrm{H}_{50} \mathrm{~N}_{2} \mathrm{O}_{6} \mathrm{~S}$ & 68.28 & 7.74 & 4.30 & 68.23 & 7.80 & 4.25 \\
& $\mathrm{C}_{37} \mathrm{H}_{50} \mathrm{~N}_{2} \mathrm{O}_{6} \mathrm{~S}$ & 68.28 & 7.74 & 4.30 & 68.30 & 7.80 & 4.28 \\
\hline
\end{tabular}

